\title{
Title: Resumption of injecting drug use following release from prison in Australia
}

Winter, RJ. ${ }^{1,2}$ Young, JT. ${ }^{3,4,5}$ Stoové, M. ${ }^{1,2}$ Agius, PA. ${ }^{1,2,6}$ Hellard, ME. ${ }^{1,2}$ Kinner, SA ${ }^{2,3,7,8,9}$

${ }^{1}$ Centre for Population Health, Burnet Institute, Melbourne, Victoria, Australia

${ }^{2}$ School of Public Health and Preventive Medicine, Monash University, Victoria, Australia

${ }^{3}$ Melbourne School of Population and Global Health, The University of Melbourne, Victoria, Australia

${ }^{4}$ Centre for Health Services Research, School of Population Health, The University of Western Australia, Western Australia, Australia

${ }^{5}$ National Drug Research Institute, Curtin University, Perth, Western Australia, Australia

${ }^{6}$ Judith Lumley Centre, La Trobe University, Melbourne, Victoria, Australia

${ }^{7}$ Griffith Criminology Institute \& Menzies Health Institute Queensland, Griffith University, Queensland, Australia

${ }^{8}$ Mater Research Institute, University of Queensland, Queensland, Australia

${ }^{9}$ Centre for Adolescent Health, Murdoch Children's Research Institute, Victoria, Australia

\section{Corresponding author}

Rebecca Winter

Centre for Population Health, Burnet Institute

GPO Box 2284

Melbourne

Victoria 3001

Australia

rwinter@burnet.edu.au

$\mathrm{Ph}:+61385062328$

Word count (manuscript, not including Abstract, Figures, Tables and References): 3,861

This is the accepted version of the following article: Winter RJ, Young JT, Stoové M, Agius PA, Hellard ME, Kinner SA. Resumption of injecting drug use following release from prison in Australia. Drug Alcohol Depend. 2016;168:104111., which has been published in final form at DOI:10.1016/j.drugalcdep.2016.08.640 


\section{$\underline{\text { Abstract }}$}

Introduction: Ex-prisoners with a history of injecting drug use (IDU) experience disproportionate drug-related harm. Rapid resumption of substance use following prison release is common and evidenced in high rates of overdose mortality. However, few studies have documented the rate of IDU resumption following prison release or identified risk factors for relapse.

Methods: Structured interviews were conducted with 533 adults with a history of IDU in Queensland, Australia prior to release from prison and approximately 1, 3 and 6 months postrelease. Incidence of self-reported IDU resumption was calculated overall and for each follow-up interval. Risk factors associated with time to resumption of IDU were estimated using discrete-time survival analysis.

Results: IDU resumption was reported by $41 \%$ of participants during a median of 98 days of followup (IQR=94-121), an overall crude incidence of 1.06 per person-year. The highest rate was observed in the first month (23\%; crude incidence 2.24 per person-year). In adjusted discrete-time survival analyses, being unemployed at the previous interview (AHR=1.59; 95\% $\mathrm{Cl}: 1.10-2.30)$, shorter incarceration ( $\leq 90$ days vs. $>365$ days; $\mathrm{AHR}=2.20 ; 95 \% \mathrm{Cl}: 1.33-3.65$ ), and IDU during the index incarceration (AHR=2.80; 95\% Cl:1.92-4.09) were significantly associated with time to IDU resumption; parole was protective (AHR=0.66; 95\%Cl:0.47-0.92).

Conclusions: Evidence-based efforts to prevent IDU in prison and IDU resumption after release are important for both prisoner and public health. Enhancing opportunities for employment and capitalising on the short-term benefits of parole for ex-prisoners may delay resumption of IDU after release from prison. These strategies should complement rather than replace harm reduction efforts for this high-risk population.

Abstract word count: 250

Key words: Prisoners, Drug Users, Survival Analysis, Follow-up Studies, Incidence 


\section{Introduction}

Globally, illicit and injecting drug users are over-represented in correctional settings (Fazel et al., 2006). In Australia, around two-thirds of prisoners report illicit drug use in the year prior to imprisonment and an estimated $45 \%$ have a history of injecting drug use (IDU) (Australian Institute of Health and Welfare, 2015). Research findings show that imprisonment often fails to have any long-term beneficial impact on substance use trajectories, instead serving as a temporary interruption to use (DeBeck et al., 2009; Evans et al., 2009), as substance use is usually reduced or ceased during imprisonment. Incarceration is often considered an opportunity for the rehabilitation of prisoners with a history of problematic substance use. However, incarceration may increase the risk of return to IDU among those who cease injection prior to imprisonment (Genberg et al., 2015), and decrease the likelihood of IDU cessation following imprisonment in the longer term (Bruneau et al., 2004; DeBeck et al., 2009; Evans et al., 2009). Incarceration may also decrease access to the tools that support cessation, such as community participation (DeBeck et al., 2009). Collectively, these findings question the individual and societal benefits of incarcerating individuals whose non-violent offences are attributable to substance use.

Prisoners also experience high rates of co-occurring health and socio-economic disadvantage prior to incarceration including unemployment, housing instability, mental disorder, infectious and chronic disease and other social and health disparities (Baldry et al., 2006; Fazel and Baillargeon, 2011; Larney et al., 2013; Cutcher et al., 2014). Among those with a history of IDU, these factors may increase the likelihood of resumption of IDU following release from prison (Binswanger et al., 2012). In addition, the difficulties of community integration after release from prison (Mallik-Kane and Visher, 2008; Visher et al., 2011), and the challenges posed by return to drug using networks (Binswanger et al., 2012; Malouf et al., 2012) may exacerbate the risk of IDU resumption. Release from prison has been associated with engaging in high-risk IDU behaviour, with some studies reporting a rapid return to substance use following release from prison (Shewan et al., 2001; Kinner, 2006; Evans et al., 2009; Milloy et al., 2009; Binswanger et al., 2012), unemployment (Visher et al., 2011), homelessness (Evans et al., 2009), disruption or restricted access to drug (Dolan et al., 2005; Stallwitz and Stöver, 2007; Fu et al., 2013) and other medical treatment programs (Milloy et al., 2011), and recidivism (Håkansson and Berglund, 2012; Kirwan et al., 2015). For ex-prisoners, IDU carries a high risk of fatal (Merrall et al., 2010) and non-fatal (Winter et al., 2015) overdose, infectious disease acquisition and transmission (Dolan et al., 2005), and other poor health and social outcomes (Kinner, 2006; Mallik-Kane and Visher, 2008; Swan, 2015). There is increasingly compelling evidence that improving health outcomes for ex-prisoners has considerable individual and societal benefits (Kinner and Wang, 2014). 
Longitudinal studies examining post-release health outcomes are scarce and often suffer from high rates of attrition, potentially resulting in biased estimation of substance use and other health and social outcomes. Critically, there is a gap in the literature on the rate of return to IDU following release from prison, and the factors associated with accelerated drug use resumption trajectories. It is important to establish the rate of IDU resumption to help tailor appropriate interventions and time their delivery accordingly. It is well established that the risk of overdose mortality is greatest in the first few weeks following prison release (Merrall et al., 2010), indicating that resumption of risky substance use in the community occurs swiftly for at least a subset of exprisoners. However, the relationship between release from prison and IDU resumption is dynamic and complex; many social, structural and interpersonal factors may influence return to IDU and the rate at which it occurs. In this study, we measured the rate of IDU resumption following release from prison in a cohort of ex-prisoners recruited in the weeks preceding release from custody in Queensland, Australia, and identified factors associated with time to IDU resumption. 


\section{Methods}

\subsection{Study design and setting}

The Passports study was a multi-site, single-blinded, randomised controlled trial of a casemanagement re-entry intervention for sentenced adult prisoners in the state of Queensland, Australia. The study methods are described in detail elsewhere (Kinner et al., 2013). Baseline interviews were conducted within six weeks of expected release from prison and before randomisation in the seven prisons from which the majority of sentenced prisoners in the State were released. Participants were randomised to receive either usual care or a transitionalintervention that included individualised case-management in the first four weeks following release (Kinner et al., 2013). Follow-up interviews occurred approximately 1 (FU1), 3 (FU2) and 6 months (to a maximum of 12 months) (FU3) after release from prison.

\subsection{Participants}

Prisoners due to be discharged from selected prisons from August 2008 to July 2010 were identified through correctional records and screened for eligibility. Eligibility criteria included (1) expected release within six weeks of interview, (2) sentenced (i.e. not pre-trial detention), (3) imprisoned for at least four weeks, and (4) able to give informed consent. Researchers not affiliated with correctional authorities explained the study and supplied a plain-language information sheet; participants provided written informed consent to participate. Of 1,665 prisoners eligible and approached, 1,325 (80\%) consented to participate and completed a pre-release interview (Kinner et al., 2013). By key demographic and criminal justice indicators, participants were broadly representative of all persons released from prison in Queensland during the recruitment period, with the exception that women were intentionally oversampled to allow adequate numbers for sexstratified analyses (Kinner et al., 2013).

Pre-release data were collected via face-to-face, researcher-administered structured questionnaires, typically taking 60-90 minutes to complete. Follow-up interviews were conducted by telephone in the community, or -- for participants who had been reimprisoned -- in prison either by telephone or face-to-face. Participants who did not report a lifetime history of IDU or were released more than eight weeks after their baseline interview were excluded from the analyses presented here.

\subsection{Measures}


The primary outcome measure was self-reported IDU resumption in the community, following release from prison. Instances of IDU that occurred during subsequent episodes of incarceration were excluded in these analyses. At baseline, participants were asked about their lifetime IDU history (ever vs. never), and at follow-up participants were asked about injection of specific drugs since release or most recent interview. The first injection -- of cocaine, amphetamines, heroin, or other opioids -- in the community following index incarceration was dichotomised into a single variable reflecting IDU resumption (yes/no) for each follow-up interval. The types of drugs injected and frequency of injection at each follow-up interview (number of days injected in the past 28 days) was recorded for descriptive purposes.

Selection of variables potentially correlated with IDU resumption following release were informed by the literature (Butzin et al., 2005; Kinner, 2006; Mallik-Kane and Visher, 2008; Binswanger et al., 2012; Malouf et al., 2012; Genberg et al., 2015) and obtained from baseline and follow-up surveys, and from medical and administrative records supplied by Queensland Corrective Services (QCS). Socio-demographic variables at baseline included age, sex, Indigenous status, and years of schooling $(<10 / 10+$ years). Baseline measures of mental health included self-reported lifetime diagnosis of mental illness (yes/no), and psychological distress in the 4 weeks prior to interview, measured by the Kessler 10 (K10) (low/medium vs. high/very high distress)(Andrews and Slade, 2001). Participants also reported the number of visits received in prison in the preceding 4 weeks as a proxy for community social support (0/1+ visits). Self-reported IDU during the current (index) episode of incarceration (yes/no) was also recorded.

Employment status (unemployed vs. employed) and accommodation status (unstable vs. stable) were measured at baseline (reflecting status before incarceration) and at each follow-up interview; these factors were operationalised as time-varying exposures. Participants' prison reception and release dates were supplied by QCS and were linked deterministically using the QCS unique identification number; from these data we categorised index incarceration length ( $\leq 90$ days, 91-365 days, $>365$ days). Using the index release, re-incarceration data and follow-up interview dates, we also calculated each participant's time at risk of return to IDU in the community following index release. Participants' parole status after index release (yes/no) and previous incarceration history (yes/no) were also obtained from QCS records.

\subsection{Data analysis}

Means and proportions were calculated for key baseline exposure variables. Pearson chisquare tests of independence and t-tests were used to explore differences in baseline characteristics 
between participants who did and did not complete at least one follow-up to assess attrition bias. The crude incidence of IDU resumption per person-year was calculated for the sample overall and by follow-up period, by dividing the observed IDU events by the total person-years contributed during observation periods. Observation time commenced on the date of prison release and was censored on the date of the first follow-up where IDU was reported, or the last completed interview (if relapse to IDU was not reported). Since the outcome of interest was IDU resumption in the community, time spent re-imprisoned during follow-up was subtracted from the time at risk for each follow-up interval. For crude incidence estimation, because the actual date of IDU was not collected, resumption was assumed to have occurred at the midpoint between interviews where IDU status was reported to change, or the midpoint between release and first follow-up. 95\% confidence intervals around incidence estimates were calculated assuming a Poisson distribution.

Unadjusted and adjusted discrete-time hazard ratios with 95\% confidence intervals (95\% Cls) were calculated using a generalised linear model specifying a binomial distribution and complementary log-log link function on person-record interview data - approximating Cox proportional hazards regression in continuous time (Allison, 2014). A discrete-time survival approach was used as the precise date of IDU resumption was unknown. Discrete-time model estimates were offset for the log of follow-up interval duration (minus any time spent reimprisoned) to account for variability in exposure between interviews, and dummy indicators for each interview were specified to permit variation in baseline hazard across interviews. Discrete-time by factor interaction terms were estimated in modelling to explore time-specific differences in effects of exposures (i.e. testing the proportional hazards assumption). Significant interaction terms $(p<0.05)$ were examined using post-estimation contrast tests of the predicted marginal means from the adjusted model, comparing each category of the factor variable at each follow-up interval, respectively. Sidak correction was applied to adjust for multiple post-estimation contrast comparisons (Šidák, 1967).

Socio-demographic and other variables measured only at baseline were modelled as timeinvariant exposures. Variables measured across interviews (accommodation and employment status) were modelled as lagged (one interview) time-varying covariates to ensure that these factors preceded the outcome of interest in modelling. Wald tests were applied to assess goodness of fit: variables which improved model fit were retained in the final discrete-time survival model. Statistical significance was defined at the $5 \%$ level in all analyses except in building the multivariate discretetime survival model, where variables which improved model fit were selected at the $10 \%$ level. Robust standard errors were specified. All analyses were performed using Stata version 14.1 (Stata Corp, Texas, USA). 


\subsection{Ethics}

The research protocol was approved by The University of Queensland's Behavioural and Social Sciences Ethical Review Committee and Queensland Corrective Services Research Committee. 


\section{Results}

\subsection{Sample characteristics}

A total of 533 eligible participants completed at least one follow-up interview (Figure 1) and were included in analyses; 458 (77.1\%) completed FU1 (median days since release=33; IQR=31-38), 434 (73.1\%) completed FU2 (median days=98; IQR=94-121) and 404 (68.0\%) completed FU3 (median days=207; IQR=187-247). Compared with those included in the analyses, participants lost to followup ( $n=98)$ were significantly less likely to have been released on parole from their index incarceration $\left(\chi^{2}=12.68, d f=1 ; p<0.001\right)$ or to have received visits from family or friends in the 4 weeks prior to their pre-release interview $\left(\chi^{2}=7.30, d f=1 ; p<0.01\right)$.

[insert Figure 1 about here]

Table 1 presents sample baseline characteristics. Twenty-four percent of participants were female and $21 \%$ were Indigenous. The mean age was $31( \pm 7.7)$ years. Most participants $(81 \%)$ had a prior history of imprisonment, and $20 \%$ reported injecting drugs during their current episode of incarceration.

[insert Table 1 about here]

\subsection{IDU resumption following prison release}

Of 533 participants who reported a history of IDU and completed at least one follow-up interview, 217 (40.7\%) reported IDU over 205.2 person-years of observation in the community following release from prison; an overall annual crude incidence of 1.06 per person-year (Table 2). The highest rate of IDU resumption was observed in the first follow-up period; 104/458 participants (23\%) reported IDU by the first follow-up interview (median days since release=33; IQR=31-38), a crude annual incidence of 2.24 per person-year.

[insert Table 2 about here] 
Methamphetamine was the most commonly reported drug injected over the period of observation ( $n=170 ; 78 \%$ ), followed by opioids, including heroin ( $n=118 ; 54 \%)$. Thirty-three percent of those who reported IDU resumption $(n=71)$ reported injecting both methamphetamines and opioids during follow-up. Among participants reinterviewed in the community who reported IDU, the median number of days injected in the 28 days prior to interview was 5.5 (IQR=2-16) for methamphetamine and 7.5 (IQR=3-21) for opioids, including heroin.

\subsection{Correlates of IDU resumption}

A number of baseline variables that did not improve model fit were excluded from the discrete-time survival analyses: randomisation to the Passports intervention, years of schooling, K10 psychological distress classification and social visits in prison. In unadjusted analyses, unstable accommodation and unemployment at the previous interview, having a previous history of imprisonment, and IDU during the index incarceration were associated with an increased risk of IDU resumption in the community following release from prison (Table 3 ).

In adjusted analyses, being unemployed at the previous interview, incarceration $\leq 90$ days (compared with $>365$ days), and having injected drugs during the index incarceration were significantly associated with resumption of IDU in the community. Being released on parole from the index incarceration was protective against IDU resumption.

[insert Table 3 about here]

Using post-estimation Wald tests, age (Wald=8.61, $d f=1 ; p=0.01$ ), incarceration length (Wald $=11.13, d f=4 ; p=0.03$ ), and parole status (Wald=6.24, $d f=2 ; p=0.04$ ) were identified as discretetime dependent, and there was evidence of joint-effect heterogeneity across discrete-time. The Sidak-adjusted model indicated that, during the first follow-up interval, participants who spent 90 days or less in custody had greater risk of IDU resumption than did those who spent 91-365 days (AHR=2.67; 95\% $\mathrm{Cl}: 1.46-4.88$ ) or longer than 365 days (AHR=3.67; 95\% $\mathrm{Cl}: 1.57-8.60)$ in custody. There was no association between time spent imprisoned and reporting IDU at subsequent followup intervals. Participants released on parole from their index incarceration had lower risk of IDU 
resumption in the community during the first follow-up interval than did those not released on parole (AHR=2.10; 95\% $\mathrm{Cl} 1.23-3.61)$, but this effect decayed over time. Ex-prisoners aged less than 40 years were at significantly greater risk of IDU resumption during the first follow-up interval, than at subsequent follow-up intervals. No difference in IDU resumption risk over time was observed for ex-prisoners aged 40 years and older. The predicted marginal probabilities for IDU resumption by incarceration length, age, parole status and discrete-time interval are displayed in Figure 2.

[insert Figure 2 about here] 


\section{Discussion}

Our results show that prisoners with a history of IDU often recommence IDU in the community soon after release from prison with nearly one quarter of participants reporting IDU within one month following release and two fifths reporting IDU over approximately 6 months following release. Of those who reported IDU in the community after release, a significant proportion never ceased IDU while imprisoned, and this was the strongest predictor of time to IDU resumption following release.

To our knowledge, only one other longitudinal study has examined time to relapse to IDU and non-IDU following prison release. Butzin and colleagues (Butzin et al., 2005) compared drug use relapse rates in ex-prisoners who participated in a transitional residential therapeutic community combined with work release treatment program $(n=945)$ with untreated controls $(n=374)$. Time to relapse in the treated group was significantly longer than in controls (mean 29 vs. 13 months), with $10 \%$ and $32 \%$ of each group relapsing by 5 years respectively. While the study suffered from selection bias - only approved prisoners were selected for the program and priority was given to those completing in-prison treatment programs - it is worth noting that relapse in both the treated and non-treated groups took considerably longer than in the current study. All participants in our study had a history of IDU, while those followed by Butzin et al were a mixture of IDU and non-IDU and the results were not disaggregated by IDU status. It is possible that those with an IDU history in our study had greater severity of drug dependence prior to incarceration than did non-IDU drug users (Novak and Kral, 2011), which may partly explain the more rapid return to IDU observed. Additionally, few participants in our study reported any form of drug treatment after release from prison.

Our results suggest that employment is a significant protective factor against IDU resumption after release from prison. Our findings are consistent with other research showing that access to mechanisms that support social stability and engagement (such as employment (Visher and Travis, 2003; Visher et al., 2011), secure housing (Baldry et al., 2003), and healthcare (Luther et al., 2011)) are critical factors in achieving positive post-release trajectories. However, access to these supports is inadequate for many former prisoners, particularly when they return to impoverished communities (Luther et al., 2011). Frequent incarceration and rapid turnover may also contribute to instability: participants in our study who were incarcerated for 90 days or less were more likely to return to IDU than were those incarcerated for longer periods. In Australia, short prison sentences are common for non-violent drug-related crimes (Australian Bureau of Statistics, 2015), so it is possible that participants imprisoned for non-violent drug-related crimes were over-represented in 
the group who spent less time in prison. Young people are particularly over-represented in the cohort that cycle rapidly in and out of prison in Australia (Australian Bureau of Statistics, 2015). In our sample, the $<40$ year age groups exhibited significantly increased risk of IDU resumption at FU1 compared to the risk of those the same age at either FU2 and FU36, while those over 40 years had no difference in risk of IDU resumption over the follow-up period.

Among our sample, being released from prison on parole was protective against IDU resumption in the community in the first month following release. Parole is the completion of a sentence under supervision in the community, after a period of time served in prison. Specific parole conditions must be met, and a breach of these conditions may result in reimprisonment for some or all of the remainder of the sentence. For those who have committed drug-related crimes, a common parole condition is regular drug testing through urinalysis. Our findings suggest that parole supervision may be protective against return to IDU, at least in the first month after release from prison. The requirement to submit to drug testing combined with the threat of reimprisonment may have served as a short term deterrent to IDU in the community. Parole may make an initial positive contribution to community integration and prevent IDU resumption, however there is little evidence to support positive long term outcomes once this supervision is withdrawn (Broadhurst, 1990; Halsey, 2010).

IDU during the index incarceration was strongly associated with a rapid resumption of IDU in the community in our sample. Although it is likely that IDU in prison occurred at a reduced frequency, IDU in prison carries with it particularly increased risk of infectious disease transmission due to the high background prevalence of infection (Dolan et al., 2016), and the lack of sterile injecting equipment and other harm reduction measures. Prison presents an opportunity to intervene and provide assistance with reducing the demand for illicit drugs, and this can complement supply reduction efforts. Opioid substitution treatment (OST) is one effective intervention to reduce demand and harm related to opioid injecting. Notably, Queensland is the only jurisdiction in Australia where OST is not routinely available for prisoners. In other parts of Australia, OST is offered during imprisonment and in at least one jurisdiction, dispensing of OST is subsidised for 4 weeks following release (Justice Health, 2015). Rates of IDU resumption may be lower in these jurisdictions, although further research is required. Providing in-prison OST may reduce IDU while incarcerated, and reduce infectious disease risk through syringe sharing (Larney, 2010; Hedrich et al., 2012) and inprison mortality (Larney et al., 2014). However, the benefits of in-prison OST alone may be shortlived without post-release retention in OST which has been shown to be associated with reduced mortality (Degenhardt et al., 2014) and recidivism (Dolan et al., 2005; Larney et al., 2012). A number of studies reporting on opioid use after release from prison found significant reductions in heroin 
use among OST recipients compared to controls during 1-12 month follow-up periods (Hedrich et al., 2012).

While it is important to continue to implement proven strategies to reduce the harms related to opioid use, the most commonly reported drug injected in our sample was methamphetamine. This corroborates other recent research showing that methamphetamine is now the most commonly reported drug used among Australian prison entrants (Australian Institute of Health and Welfare, 2015). Given the limitations of existing treatments for methamphetamine dependence (Lee et al., 2008), this presents new and significant challenges for harm reduction and community re-entry policy.

\subsection{Limitations}

Our study had at least five important limitations. First, we cannot guarantee that the cohort was representative of all prisoners transitioning from prison to the community in Queensland despite a representative sampling approach, high recruitment fraction, relatively low attrition, and good evidence that participants were representative of released prisoners across the State on demographic and criminal justice measures (Kinner et al., 2013). Participants may have differed from non-participants on other, unmeasured, variables. Furthermore, it is unclear to what extent these findings can be generalised to other settings in Australia or elsewhere. Replication in other settings, particularly those where OST is available for prisoners, is required. Second, we selected participants on the basis of lifetime IDU history and were not able to confirm that these individuals were injecting immediately prior to incarceration. As such, our estimates of IDU resumption among recently active injectors are likely conservative. However, we have no a priori reason to suspect that our estimates of association (predictors of IDU resumption) are biased on the basis of our inclusion criteria of lifetime IDU. Third, our outcome measure was any reported IDU during follow-up, such that those who injected even once after release from prison were treated the same as those who reported more frequent injecting. Although the latter group might be expected to be at greater risk of drug-related harm, even infrequent IDU carries with it the risk of acute harms such as overdose (Brugal et al., 2002) and the transmission of infectious disease (Hagan et al., 2004). Fourth, although we included an offset for the discrete-time survival model, in the estimation of crude incidence, instances of IDU were ascribed at the midpoint of the period preceding the follow-up interview during which IDU was first reported. As such, we may have under-estimated the extent to which IDU resumption was concentrated in the first month post-release. For example, for individuals who missed the first timed follow-up interview (median 33 days post-release) but reported IDU 'since last interview' at the second follow-up (median 98 days post-release), the resumption was deemed to 
have occurred by follow-up two, although it may have occurred within the first month post-release. Finally, readers should note that the discrete-time survival analyses undertaken are based on the common assumption that random censoring (i.e. loss-to-follow-up) in the cohort is, conditional on covariates, non-informative (i.e. censoring at a particular time is independent of a participant's hazard at that time). Potential bias may result where this assumption is not met, for example if those who were randomly censored in the study due to death or returning to prison exhibited greater hazard of community IDU resumption at the time.

\section{Conclusion}

Rapid resumption of IDU following release from prison is consistent with the view that incarceration often represents an interruption in drug-using careers and offers only limited rehabilitation opportunities for drug using offenders. Given growing evidence of poor health and criminal justice outcomes for people who inject drugs after release from custody (Kinner, 2006; Mallik-Kane and Visher, 2008) these findings highlight the critical importance of increased efforts to limit IDU in prison and IDU resumption after release from prison. Improving opportunities for employment and capitalising on the short-term benefits of parole for ex-prisoners may delay resumption of IDU after release from prison, providing further opportunities to reduce harms and improve community participation. 


\section{References}

Allison, P., 2014. Event history and survival analysis. Thousand Oaks, CA, Sage University Papers Series on Quantitative Applications in the Social Sciences.

Andrews, G. and Slade, T., 2001. Interpreting scores on the Kessler Psychological Distress Scale (K10). Aust N Z J Public Health, 25, 494-497.

Australian Bureau of Statistics, 2015. Prisoners in Australia. Cat no.4517.0, ABS.

Australian Institute of Health and Welfare, 2015. The health of Australia's prisoners 2015. Canberra, Cat. no. PHE 207. AlHW.

Baldry, E., McDonnell, D., Maplestone, P. and Peeters, M., 2003. Ex-prisoners and accommodation: what bearing do different forms of housing have on social reintegration? Australian Housing and Urban Research Institute.

Baldry, E., McDonnell, D., Maplestone, P. and Peeters, M., 2006. Ex-prisoners, homelessness and the state in Australia. Australian \& New Zealand Journal of Criminology, 39, 20-33.

Binswanger, I.A., Nowels, C., Corsi, K.F., Glanz, J., Long, J., Booth, R.E. and Steiner, J.F., 2012. Return to drug use and overdose after release from prison: a qualitative study of risk and protective factors. Addiction Science \& Clinical Practice, 7, 3 DOI: 10.1186/1940-0640-7-3.

Broadhurst, R., 1990. Evaluating imprisonment and parole: survival rates or failure rates? Australian Institute of Criminology.

Brugal, M.T., Barrio, G., Fuente, L.D.L., Regidor, E., Royuela, L. and Suelves, J.M., 2002. Factors associated with non-fatal heroin overdose: assessing the effect of frequency and route of heroin administration. Addiction, 97, 319-327.

Bruneau, J., Brogly, S.B., Tyndall, M.W., Lamothe, F. and Franco, E.L., 2004. Intensity of drug injection as a determinant of sustained injection cessation among chronic drug users: the interface with social factors and service utilization. Addiction, 99, 727-737.

Butzin, C.A., Martin, S.S. and Inciardi, J.A., 2005. Treatment during transition from prison to community and subsequent illicit drug use. J Subst Abuse Treat, 28, 351-358.

Cutcher, Z., Degenhardt, L., Alati, R. and Kinner, S.A., 2014. Poor health and social outcomes for exprisoners with a history of mental disorder: a longitudinal study. Aust N Z J Public Health, 38, 424-429.

DeBeck, K., Kerr, T., Li, K., Milloy, M.J., Montaner, J. and Wood, E., 2009. Incarceration and drug use patterns among a cohort of injection drug users. Addiction, 104, 69-76.

Degenhardt, L., Larney, S., Kimber, J., Gisev, N., Farrell, M., Dobbins, T., Weatherburn, D.J., Gibson, A., Mattick, R. and Butler, T., 2014. The impact of opioid substitution therapy on mortality post-release from prison: retrospective data linkage study. Addiction, 109, 1306-1317.

Dolan, K., Wirtz, A., Moazen, B., Galvani, A., Ndeffo-mbah, M., Kinner, S., Courtney, R., McKee, M., Amon, J., Hellard, M., Altice, R. and Beyrer, C., 2016. Global burden of HIV, viral hepatitis and tuberculosis among prisoners and detainees. The Lancet.

Dolan, K.A., Shearer, J., White, B., Zhou, J., Kaldor, J. and Wodak, A.D., 2005. Four-year follow-up of imprisoned male heroin users and methadone treatment: mortality, re-incarceration and hepatitis C infection. Addiction, 100, 820-828.

Evans, J.L., Hahn, J.A., Lum, P.J., Stein, E.S. and Page, K., 2009. Predictors of injection drug use cessation and relapse in a prospective cohort of young injection drug users in San Francisco, CA (UFO Study). Drug Alcohol Depend, 101, 152-157.

Fazel, S., Bains, P. and Doll, H., 2006. Substance abuse and dependence in prisoners: a systematic review. Addiction, 101, 181-191.

Fazel, S. and Baillargeon, J., 2011. The health of prisoners. The Lancet, 377, 956-965.

Fu, J.J., Zaller, N.D., Yokell, M.A., Bazazi, A.R. and Rich, J.D., 2013. Forced withdrawal from methadone maintenance therapy in criminal justice settings: a critical treatment barrier in the United States. J Subst Abuse Treat, 44, 502-505.

Genberg, B., Astemborski, J., Vlahov, D., Kirk, G. and Mehta, S., 2015. Incarceration and injection drug use in Baltimore, Maryland. Addiction, 110, 1152-1159. 
Hagan, H., Thiede, H. and Des Jarlais, D.C., 2004. Hepatitis C Virus Infection Among Injection Drug Users: Survival Analysis of Time to Seroconversion. Epidemiology, 15, 543-549.

Håkansson, A. and Berglund, M., 2012. Risk factors for criminal recidivism - a prospective follow-up study in prisoners with substance abuse. BMC Psychiatry, 12 DOI: 10.1186/1471-244X-12-111.

Halsey, M., 2010. Imprisonment and prisoner re-entry in Australia. Dialectical Anthropology, 34, 545554.

Hedrich, D., Alves, P., Farrell, M., Stöver, H., Møller, L. and Mayet, S., 2012. The effectiveness of opioid maintenance treatment in prison settings: a systematic review. Addiction, 107, 501517.

Justice Health, 2015. Victorian Prison Opioid Substitution Therapy Program Guidelines. Melbourne, Justice Health, Department of Justice \& Regulation, Victorian Government.

Kinner, S.A., 2006. Continuity of health impairment and substance misuse among adult prisoners in Queensland, Australia. International Journal of Prisoner Health, 2, 101-113.

Kinner, S.A., Lennox, N., Williams, G.M., Carroll, M., Quinn, B., Boyle, F.M. and Alati, R., 2013. Randomised controlled trial of a service brokerage intervention for ex-prisoners in Australia. Contemp Clin Trials, 36, 198-206.

Kinner, S.A. and Wang, E.A., 2014. The case for improving the health of ex-prisoners. Am J Public Health, 104, 1352-1355.

Kirwan, A., Quinn, B., Winter, R., Kinner, S.A., Dietze, P. and Stoové, M., 2015. Correlates of property crime in a cohort of recently released prisoners with a history of injecting drug use. Harm Reduct J, 12, 1.

Larney, S., 2010. Does opioid substitution treatment in prisons reduce injecting-related HIV risk behaviours? A systematic review. Addiction, 105, 216-223.

Larney, S., Toson, B., Burns, L. and Dolan, K., 2012. Effect of prison-based opioid substitution treatment and post-release retention in treatment on risk of re-incarceration. Addiction, 107, 372-380.

Larney, S., Kopinski, H., Beckwith, C.G., Zaller, N.D., Jarlais, D.D., Hagan, H., Rich, J.D., van den Bergh, B.J. and Degenhardt, L., 2013. Incidence and prevalence of hepatitis $C$ in prisons and other closed settings: results of a systematic review and meta-analysis. Hepatology, 58, 1215-1224.

Larney, S., Gisev, N., Farrell, M., Dobbins, T., Burns, L., Gibson, A., Kimber, J. and Degenhardt, L., 2014. Opioid substitution therapy as a strategy to reduce deaths in prison: retrospective cohort study. BMJ Open, 4, e004666. Available at http://bmjopen.bmj.com/content/4/4/e004666.full

Lee, N.K., Lee, N.K., Rawson, R.A., Lee, N.K. and Rawson, R.A., 2008. A systematic review of cognitive and behavioural therapies for methamphetamine dependence. Drug Alcohol Rev, 27, 309-317.

Luther, J.B., Reichert, E.S., Holloway, E.D., Roth, A.M. and Aalsma, M.C., 2011. An exploration of community reentry needs and services for prisoners: A focus on care to limit return to highrisk behavior. AIDS Patient Care STDS, 25, 475-481.

Mallik-Kane, K. and Visher, C., 2008. Health and prisoner reentry: how physical, mental and substane abuse conditions shape the process of reintegration, Justice Policy Center, Urban Institute.

Malouf, E., Stuewig, J. and Tangney, J., 2012. Self-control and jail inmates' substance misuse postrelease: Mediation by friends' substance use and moderation by age. Addict Behav, 37, 11981204.

Merrall, E.L.C., Kariminia, A., Binswanger, I.A., Hobbs, M.S., Farrell, M., Marsden, J., Hutchinson, S.J. and Bird, S.M., 2010. Meta-analysis of drug-related deaths soon after release from prison. Addiction, 105, 1545-1554.

Milloy, M., Kerr, T., Buxton, J., Rhodes, T., Guillemi, S., Hogg, R., Montaner, J. and Wood, E., 2011. Dose-response effect of incarceration events on nonadherence to HIV antiretroviral therapy among injection drug users. J Infect Dis, 203, 1215-1221.

Milloy, M.J., Buxton, J., Wood, E., Li, K., Montaner, J.S. and Kerr, T., 2009. Elevated HIV risk behaviour among recently incarcerated injection drug users in a Canadian setting: a longitudinal analysis. 
BMC Public Health, 9. Available at:

http://bmcpublichealth.biomedcentral.com/articles/10.1186/1471-2458-9-156.

Novak, S.P. and Kral, A.H., 2011. Comparing Injection and Non-Injection Routes of Administration for Heroin, Methamphetamine, and Cocaine Uses in the United States. J Addict Dis, 30, 248-257.

Shewan, D., Reid, M., MacPherson, S., Davies, J.B. and Greenwood, J., 2001. Injecting risk behaviour among recently released prisoners in Edinburgh: The impact of in-prison and community drug treatment services. Legal and Criminological Psychology, 6, 19-28.

Šidák, Z., 1967. Rectangular Confidence Regions for the Means of Multivariate Normal Distributions. Journal of the American Statistical Association, 62, 626-633.

Stallwitz, A. and Stöver, H., 2007. The impact of substitution treatment in prisons-a literature review. International Journal of Drug Policy, 18, 464-474.

Swan, H., 2015. Different patterns of drug use and barriers to continuous HIV care postincarceration. Journal of Drug Issues, 45, 38-52. Available at: http://www.ncbi.nlm.nih.gov/pmc/articles/PMC4448759/

Visher, C. and Travis, J., 2003. Transitions from prison to community: understanding individual pathways. Annual Review Sociology 29, 89-113.

Visher, C.A., Debus-Sherrill, S.A. and Yahner, J., 2011. Employment After Prison: A Longitudinal Study of Former Prisoners. JQ: Justice Quarterly, 28, 698-718.

Winter, R.J., Stoové, M., Degenhardt, L., Hellard, M.E., Spelman, T., Jenkinson, R., McCarthy, D.R. and Kinner, S.A., 2015. Incidence and predictors of non-fatal drug overdose after release from prison among people who inject drugs in Queensland, Australia. Drug Alcohol Depend, 153, 43-49. 
TABLES/FIGURES:

Table 1: Cohort characteristics at baseline

\begin{tabular}{|c|c|}
\hline & $\begin{array}{l}\mathrm{N}=533 \\
\mathrm{n}(\%)^{*}\end{array}$ \\
\hline Female & $125(23.5)$ \\
\hline Indigenous & $111(20.8)$ \\
\hline Age in years (mean (SD)) & $30.7(7.7)$ \\
\hline Randomised to Passports intervention & $265(49.7)$ \\
\hline Unstable accommodation & $108(20.3)$ \\
\hline$<10$ years schooling ${ }^{a}$ & $263(49.4)$ \\
\hline Unemployed & $287(53.9)$ \\
\hline Previous imprisonment & 431 (80.9) \\
\hline \multicolumn{2}{|l|}{ Length of index incarceration } \\
\hline$\leq 90$ days & $178(33.4)$ \\
\hline $91-365$ days & $262(49.2)$ \\
\hline$>365$ days & $93(17.5)$ \\
\hline Released on parole from index incarceration & $231(43.3)$ \\
\hline Diagnosed mental illness ever & $282(52.9)$ \\
\hline Current high/very high psychological distress ${ }^{b}$ & $135(25.3)$ \\
\hline Injected drugs during index incarceration & $108(20.3)$ \\
\hline Received $\geq 1$ visit in prison during past 4 weeks & $236(44.3)$ \\
\hline
\end{tabular}

\footnotetext{
${ }^{a}$ Missing $=1$

${ }^{\mathbf{b}}$ Versus low/medium distress, measured by the Kessler 10

* Percentages are rounded
} 
Table 2: Conditional annual crude incidence rate and 95\% confidence intervals per follow-up period for IDU resumption in the community after prison release.

\begin{tabular}{lccccc}
\hline Time interval & $\mathbf{n}$ & $\begin{array}{c}\text { Number of } \\
\text { participants } \\
\text { who resumed } \\
\text { IDU }\end{array}$ & $\begin{array}{c}\text { Follow-up time } \\
\text { (PY) }\end{array}$ & $\begin{array}{c}\text { Mean annual IR } \\
\text { per person year }\end{array}$ & $\mathbf{9 5 \% ~ C l}$ \\
\hline Release - FU1 & 458 & 104 & 46.46 & 2.24 & $\mathbf{1 . 8 3 - 2 . 7 1}$ \\
FU1 - FU2 & 358 & 65 & 70.09 & 0.93 & $\mathbf{0 . 7 2 - 1 . 1 8}$ \\
FU2 - FU3 & 295 & 48 & 88.61 & 0.54 & $\mathbf{0 . 4 0 - 0 . 7 2}$ \\
\hline Total & $\mathbf{5 3 3}$ & $\mathbf{2 1 7}$ & $\mathbf{2 0 5 . 1 7}$ & $\mathbf{1 . 0 6}$ & $\mathbf{0 . 9 2 - 1 . 2 1}$ \\
\hline
\end{tabular}

NB: IR = incidence rate, $95 \% \mathrm{Cl}=95 \%$ confidence intervals, $\mathrm{PY}=$ person-years, $\mathrm{FU}=$ follow-up, IDU = injecting drug use 
Table 3: Unadjusted and adjusted hazard ratios with $95 \%$ confidence intervals and probability values for IDU resumption in the community following prison release $(\mathrm{N}=533)$

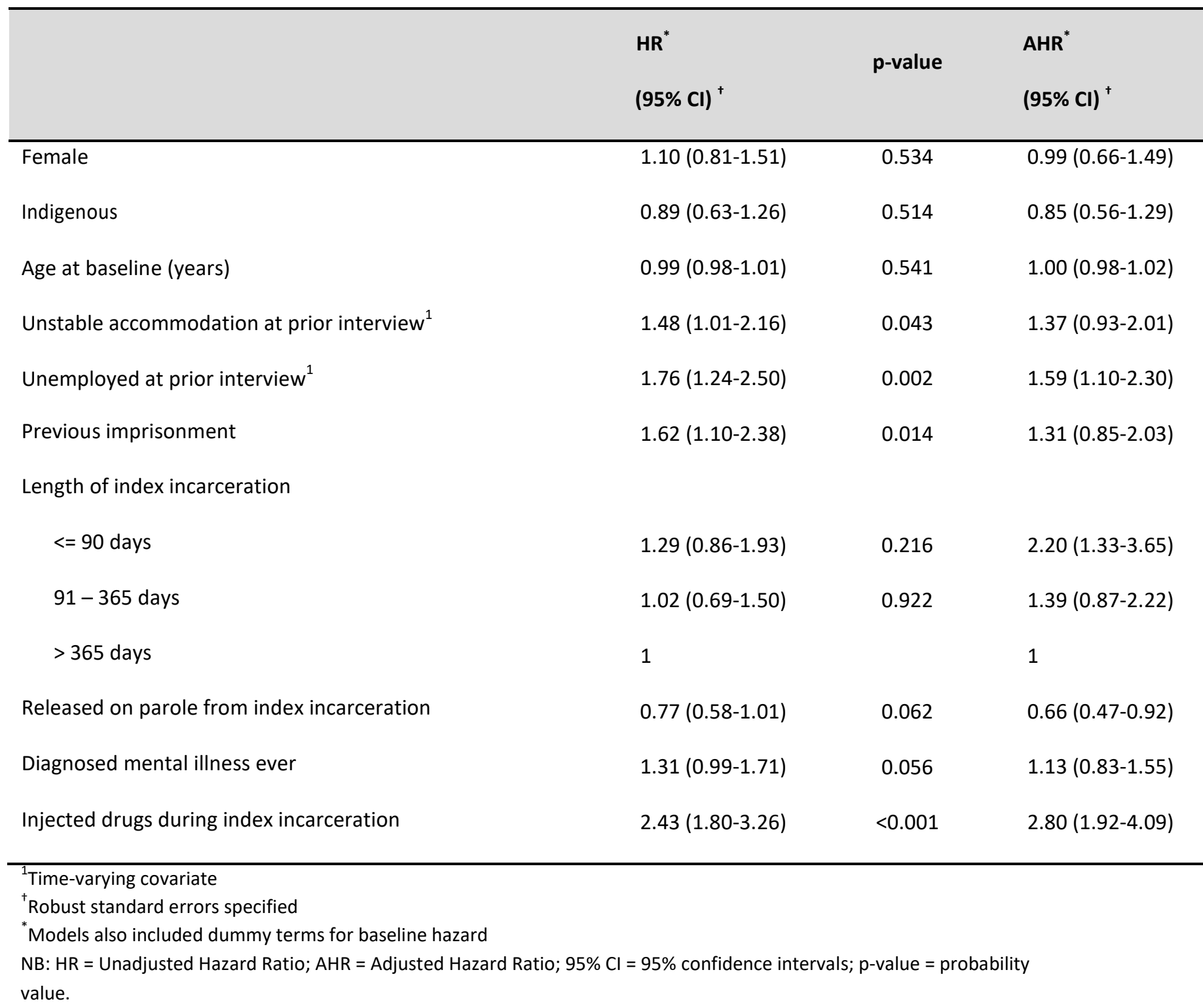




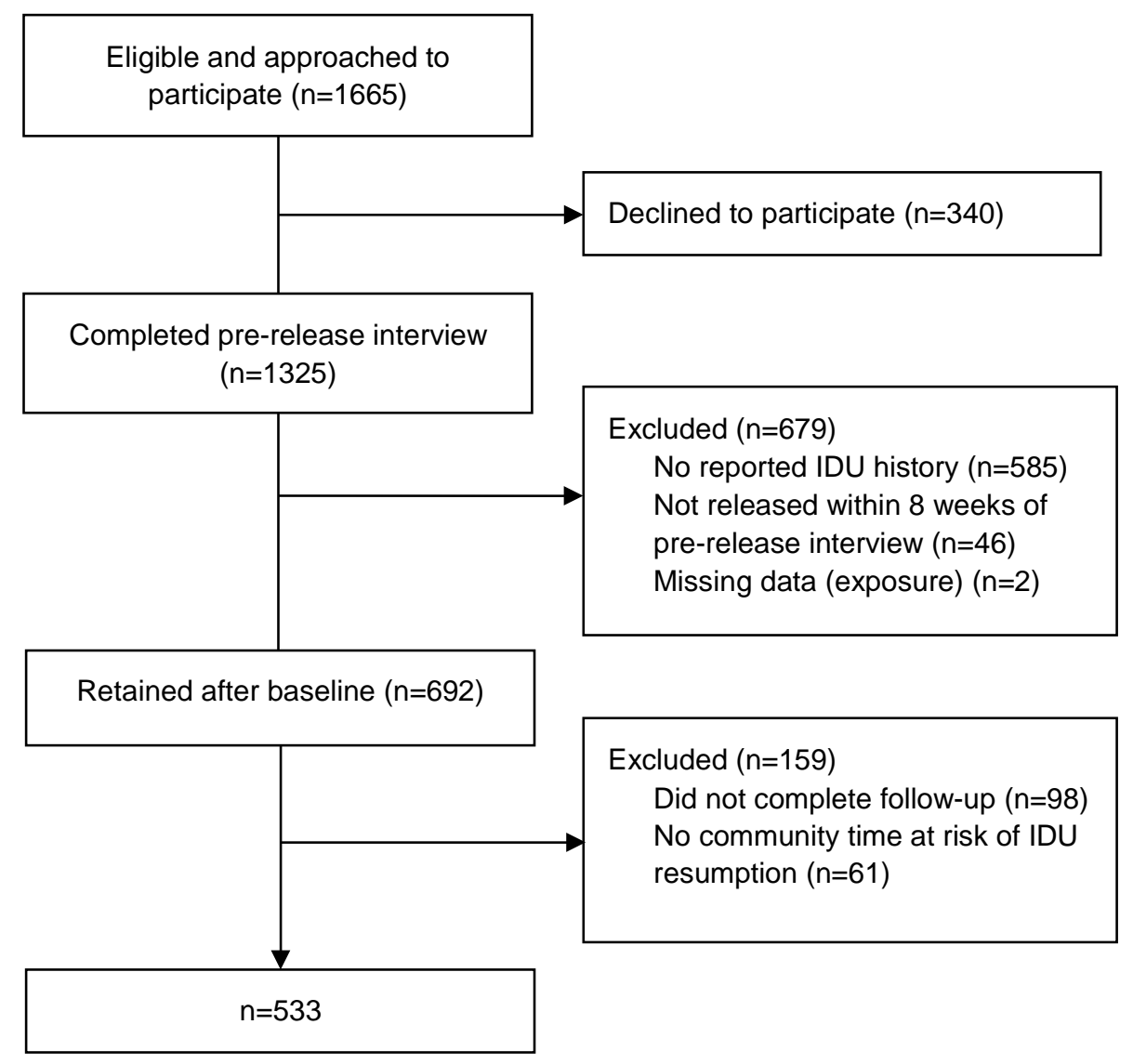

Figure 1: Participant inclusion and exclusion 

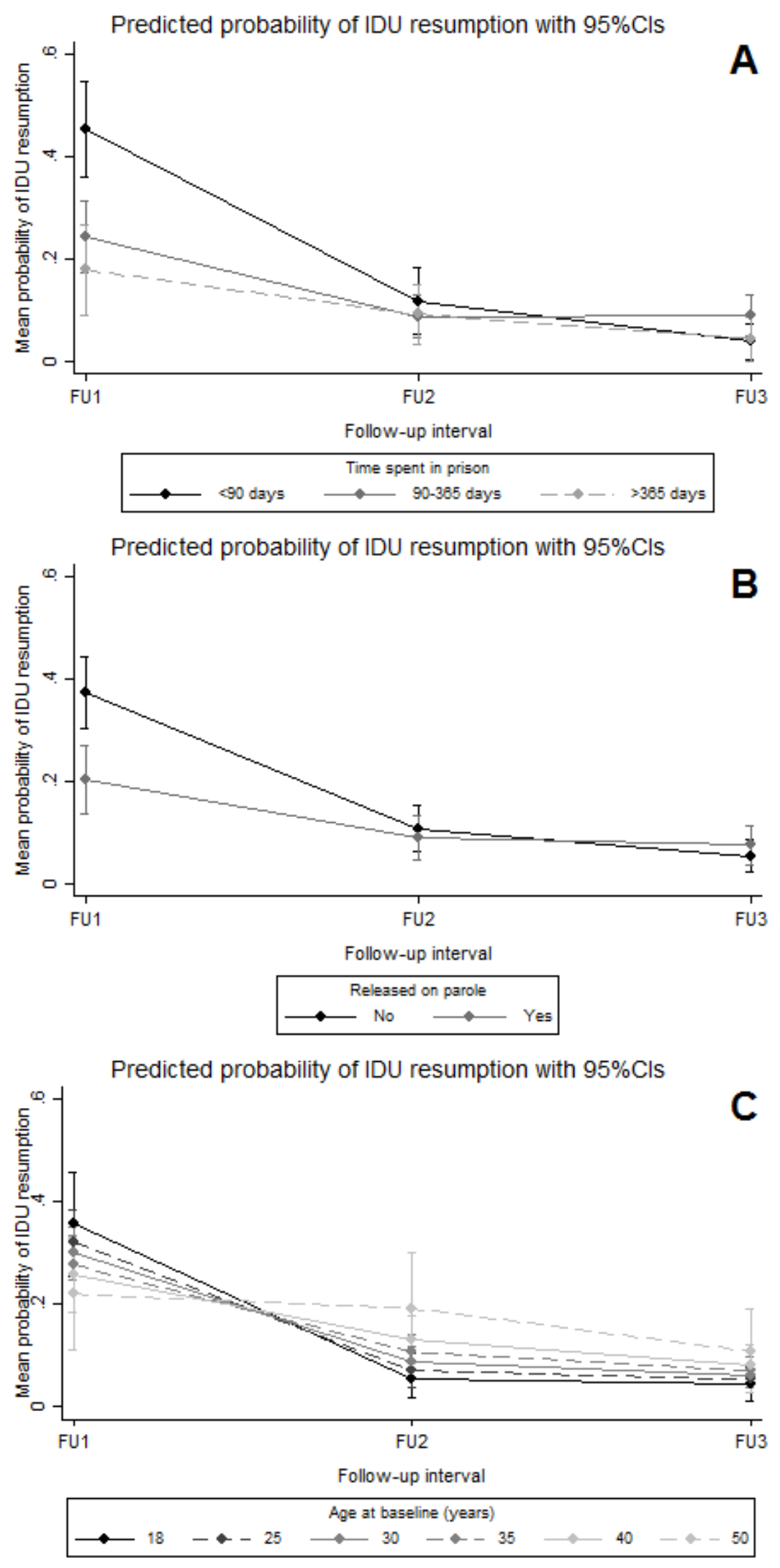

Figure 2. Predicted marginal means of injecting drug use resumption with $95 \%$ confidence intervals at each follow-up interval for time-dependent covariates. FU: Follow-up; IDU: Injecting drug use; 95\% Cl: 95\% confidence intervals 
